\title{
DECOMPOSITION NUMBERS OF $p$-SOLVABLE GROUPS ${ }^{1}$
}

\section{FORREST RICHEN}

ABSTRACT. In the character theory of finite groups one decomposes each ordinary irreducible character $\chi_{i}$ of a group into an integral linear combination of $p$-modular irreducible characters $\phi_{j}$, $\chi_{i}=\sum d_{i j} \phi_{j}$. The nonnegative integers $d_{i j}$ are called the $p$-decomposition numbers. Let $G$ be a $p$-solvable group whose $p$-Sylow subgroups are abelian. If $G / O_{p^{\prime} p}(G)$ is cyclic the $p$-decomposition numbers are $\leqq 1$. This condition is far from necessary as any group $G$ with abelian, normal $p$-Sylow subgroup $P$ with $G / P$ abelian has $p$-decomposition numbers $\leqq 1$. A result of Brauer and Nesbitt together with the first result yields the following. A group $G$ has a normal $p$-complement and abelian p-Sylow subgroups if and only if each irreducible character of $G$ is irreducible as a $p$-modular character.

A group is said to be $p$-solvable if each of its composition factors is either a $p$-group or a $p^{\prime}$-group, $p$ a prime number. Denote by $O_{p^{\prime}}(G)$ and $O_{p^{\prime} p}(G)$ the largest normal $p^{\prime}$-subgroup of a group $G$ and the inverse image of the largest normal $p$-subgroup of $G / O_{p^{\prime}}(G)$ under the natural homomorphism $G \rightarrow G / O_{p^{\prime}}(G)$ respectively. The following proposition is 1.2 .3 of [6]. If $G$ is a $p$-solvable group and $P$ is a $p$ Sylow subgroup of $G$ then the center of $P, Z(P) \subset O_{p^{\prime} p}(G)$. As a result, if $P$ is abelian $P \subset Z(P) \subset O_{p^{\prime} p}(G)$ and so $O_{p^{\prime} p}(G)=P O_{p^{\prime}}(G)$. It also follows that $\left[G: O_{p^{\prime} p}(G)\right]$ is relatively prime to $p$.

We assume knowledge of elementary character theory and recall some facts about the relationship between the ordinary and modular representations of a group $G$. References [1] and [2] will supply the details. Let $K$ be the extension of the rational numbers obtained by adjoining the $m$ th roots of 1 where $m$ is the exponent of $G . R$ denotes the valuation ring of an extension of the $p$-adic valuation to $K$ in $K$, $\rho$ denotes the maximal ideal of $R$ and $\bar{K}=R / \odot$ a field of characteristic $p$.

If $\mathrm{Z}$ is a $K$-representation of $G$, there exists a $K$-similar representation $Z^{\prime}$ such that all the entries of $Z^{\prime}(g), g \in G$, lie in $R$. By reducing the entries of $Z^{\prime}(g)$ modulo $\odot$ we get a $\bar{K}$-representation $\bar{Z}$ of $G$. This process does not determine $\bar{Z}$ uniquely, but the composition factors of $\bar{Z}$ are uniquely determined by $\bar{Z}$.

Presented to the Society, August 29, 1969; received by the editors September 15, 1969.

A MS Subject Classifications. Primary 2080; Secondary 2027.

Key Words and Phrases. p-solvable group, decomposition numbers, $p$-modular character, ordinary character.

1 This research was supported in part by NSF Grants GP 8457 and GP 11542. 
Given an irreducible $\bar{K}$-representation $F$ of $G$, one constructs a function $\phi$ on the $p$-regular elements of $G$ as follows. If $g \in G$ is $p$-regular then the eigenvalues $\left\{\bar{\delta}_{i}\right\}$ of $F(g)$ are $m^{\prime}$ roots of 1 where $\left(m^{\prime}, p\right)=1$ and $m=m^{\prime} p^{n}$. Let $\left\{\delta_{i}\right\}$ be the corresponding set of roots of unity in $K$ under the natural homomorphism $R \rightarrow \bar{K}$. Define $\phi(g)=\sum \delta_{i}$. F $\leftrightarrow \phi$ is a one-to-one correspondence between the isomorphism classes of irreducible $\bar{K}$-representations $\left\{F_{i}\right\}$ and a set of functions $\left\{\phi_{i}\right\}$ called the $p$-modular characters of $G$. The $\left\{\phi_{i}\right\}$ form a basis of the $K$ vector space of class functions from the $p$-regular elements of $G$ into $K$.

It follows from these remarks that if $Z_{i}$ is an irredubible $K$-representation of $G$ with character $\chi_{i}$, then $F_{j}$ appears as a composition factor of $\bar{Z}_{i}$ with multiplicity $d_{i j}$ where $\chi_{i \mid p-\text { regular elemonts }}=\sum d_{i j} \phi_{j}$. The matrix $D=\left(d_{i j}\right)$ is called the decomposition matrix and the nonnegative integers $d_{i j}$ are called the decomposition numbers.

It is sometimes convenient to partition the ordinary and modular characters into subsets called blocks, and when the $\chi$ 's and $\phi$ 's are indexed accordingly the decomposition matrix has block diagonal form. A submatrix on the diagonal of $D$ which corresponds to a block of ordinary and modular characters is called the decomposition matrix of that block.

THEOREM. Let $G$ be a p-solvable group whose p-Sylow subgroups are abelian. If $G / O_{p^{\prime} p}(G)$ is cyclic then the p-decomposition numbers are $\leqq 1$.

The proof is given in three steps. First, using an induction method of Fong [4] we reduce the proof to a consideration of a simpler type of group. Next we reduce the proof to a question about the ordinary characters of this type of group. Last, we use a theorem of Gallagher [5] to deal with these characters.

First observe that the hypotheses of the theorem hold for subgroups $H$ of $G$ which contain $O_{p^{\prime}}(G)$. Clearly a $p$-Sylow subgroup of $H$ is abelian. Since $O_{p^{\prime}}(G) \subset H, O_{p^{\prime}}(G) \subset O_{p^{\prime}}(H)$. We observed in the introduction that $O_{p^{\prime} p}(G)=P O_{p^{\prime}}(G)$ where $P$ is a $p$-Sylow subgroup of G. Hence

$$
\begin{aligned}
O_{p^{\prime} F}(G) \cap H & =O_{p^{\prime}}(G) P \cap H \subset O_{p^{\prime}}(H) P \cap H \\
& =O_{p^{\prime}}(H)(P \cap H) \subset O_{p^{\prime} p}(H) .
\end{aligned}
$$

Thus $H / O_{p^{\prime} p}(H)$ is a homomorphic image of $H / O_{p^{\prime} p}(G) \cap H$ $\cong H O_{p^{\prime} p}(G) / O_{p^{\prime} p}(G) \cong G / O_{p^{\prime} p}(G)$ which is cyclic. Thus $H / O_{p^{\prime} p}(H)$ is cyclic.

We use induction on $|G|$ to reduce our considerations to groups with a normal $p$-Sylow subgroup. If $O_{p^{\prime}}(G)=1$ then $G$ has a normal 
p-Sylow subgroup and a cyclic complement. If $O_{p^{\prime}}(G) \neq 1$ and $B$ is a block of $G$, then Fong's theorem 2B [4] asserts that there is a subgroup $H, O_{p^{\prime}}(G) \subset H \subset G$, and a block $B^{\prime}$ of $H$ whose decomposition matrix is identical to that of $B$. If $H \neq G$ then all the decomposition numbers for characters in $B$ are $\leqq 1$ by induction. If $H=G$, then Fong's theorem 2D gives a group $M$ with a cyclic, central $p^{\prime}$-subgroup $C$ and a block $B^{\prime \prime}$ of $M$ such that $M / C \cong G / O_{p^{\prime}}(G)$ and the decomposition matrix of $B^{\prime \prime}$ is the same as that of $B$. Clearly $M$ is $p$-solvable with an abelian $p$-Sylow subgroup $P^{\prime}$. Thus $O_{p^{\prime} p}(M)=C P^{\prime}$ $=C \times P^{\prime}$, as $C$ is central, and so $P^{\prime} \triangleleft M$. Moreover since $G / O_{p^{\prime} p}(G)$ is cyclic it follows that $M / P^{\prime} C_{G}\left(P^{\prime}\right)$ is cyclic. Thus it suffices to prove the theorem for a group $G$ with normal abelian $p$-Sylow subgroup $P$ and with $G / P C_{G}(P)$ cyclic.

We isolate the next part of the proof as a

Lemma. Suppose $G=P H$ is a finite group, $P \triangleleft G$ is the $p$-Sylow subgroup and $(|H|, p)=1$. If $\phi_{j}$ is a p-modular irreducible character of $G$, then $\phi_{j \mid I I}$ is an ordinary irreducible character of $H$. Hence if $\chi_{i}$ is an ordinary character of $G$, the equation $\chi_{i}=\sum d_{i j} \phi_{j}$ gives a decomposition of $\chi_{i \mid H}$ as a sum of irreducible characters of $H$.

Proof. Let $F$ be an irreducible $\bar{K}$-representation of $G$ with modular character $\phi$. By Clifford's theorem $[2,49.7] P \subset$ kernel of $F$ and so $F(\phi)$ can be viewed as a representation (modular character) of $G / P \cong H$. But since $p \nmid|H|$ there is a $K$-irreducible representation $\mathrm{Z}$ of $H$ such that $\overline{\mathrm{Z}}=F_{\mid H},[3,4.4] . \phi_{\mid H}$ nust be the character of $\mathrm{Z}$. The rest follows immediately.

Suppose that $G$ has a normal abelian $p$-Sylow subgroup $P, p$-complement $H$ and that $G / P C_{G}(P)$ is cyclic. By the lemma we must show that $\chi_{\mid H}$ is multiplicity free for every irreducible character $\chi$ of $G$, i.e. if $\chi_{\mid I I}=\sum a_{i} \lambda_{i}, \lambda_{i}$ irreducible characters of $H$, then $a_{i} \leqq 1$ for all $i$.

By a theorem of Gallagher [5, Theorem 7] every irreducible character of $G$ can be realized in the following way. Let $\theta$ be a character of $P$. Let $T=\left\{x \in G: \theta^{x}=\theta\right\}$ be the stability group of $\theta$ and $\theta_{1}$ an extension of $\theta$ to $T$. Let $\omega$ be a character of $T$ with $P$ in its kernel. Then $\left(\theta_{1} \omega\right)^{a}$, the induced character, is irreducible and all characters of $G$ are of this form.

Observe that $P C_{G}(P) \subset T$. Since $H P=G$, the only $(H, T)$ double coset is $H T=G$. Thus the subgroup theorem [2, 44.2] implies that $\left(\theta_{1} \omega\right)_{\mid H}^{G}=\left(\left(\theta_{1} \omega\right)_{H \cap T}\right)^{H}$.

Now $H \cap T \triangleleft H$ as $C_{I I}(P) \subset H \cap T$ and $H / C_{H}(P) \cong H / H \cap C_{G}(P)$ $\cong H C_{G}(P) / C_{G}(P)=G / C_{G}(P)$ is cyclic. It also follows that $H / H \cap T$ is cyclic. 
Suppose that $\left(\left(\theta_{1} \omega\right)_{H} \cap_{T}\right)^{H}=a_{1} \lambda_{1}+\cdots+a_{n} \lambda_{n}, \lambda_{i}$ irreducible characters of $H$. Clifford's theorem and Frobenius reciprocity imply that $\lambda_{i \mid H \cap} \cap_{T}=a_{i} \sum_{x}\left(\theta_{1} \omega\right)_{\mid H}^{x} \cap_{T}$ where $x$ runs over a set of coset representatives of $H \cap T$ in the stability group of $\theta_{1} \omega$ in $H$. But this stability group is cyclic modulo $H \cap T$ since $H / H \cap T$ is cyclic. Thus a theorem of Schur $[3,9.12]$ implies that $a_{i}=1$. This completes the proof.

This theorem is not exhaustive. In fact the following proposition covers many $p$-solvable groups with abelian $p$-Sylow subgroups which are not covered by the theorem.

Proposition. If $G$ is a finite group with normal abelian p-Sylow subgroup and abelian p-complement $H$, then all p-decomposition numbers are $\leqq 1$.

Proof. Let $\chi$ be an irreducible character of $G$. By the lemma we must show that $\chi_{\mid H}$ is multiplicity free. Now by Fröbenius reciprocity the induced character $\theta^{G}=\chi+\chi^{\prime}$ for some (reducible) character $\chi^{\prime}$ of $G$ and some irreducible character $\theta$ of $P$. Thus it suffices to show that $\theta_{i H}^{G}$ is multiplicity free for every irreducible character $\theta$ on $P$.

$P \triangleleft G$ implies that $\theta^{G}(x)=0$ for $x \in H$, and $\theta^{G}(1)=|G: P|=|H|$ as $\theta$ is linear. Therefore $\theta_{\mid H}^{G}$ is the regular character on $H$, and the regular character of an abelian group contains each of its irreducible constituents with multiplicity 1 . This proves the proposition.

On the other hand examples show that none of the hypotheses of the theorem is superfluous. In particular the hypothesis that $G / O_{p^{\prime} p}(G)$ is cyclic cannot be deleted as the following shows. Let $P$ be an abelian group of type $(3,3)$ and let $Q=\langle\alpha, \beta\rangle$ be the quaternion group of order 8. Let $Q \rightarrow \operatorname{Aut}(P)$ be a homomorphism such that $\alpha$ inverts the elements of $P$ and $\beta$ exchanges the elements of some basis of $P$. (Thus the involution $u \in Q$ acts trivially.) Let $G$ be the resulting semidirect product, and identify $P$ and $Q$ with subgroups of $G$. Let $\lambda$ be a character on $P \times\langle u\rangle$ defined as the product of the nonidentity character on $\langle u\rangle$ with a linear character on $P$ whose kernel is not fixed by $\beta$. One easily checks that $P \times\langle u\rangle$ is the stability group of $\lambda$ in $G$ and that $P \times\langle u\rangle \triangleleft G$. Thus $\lambda^{G}$ is irreducible. But $u$ is central in $G$ and so $\lambda^{G}(u)=-4$. Thus $\lambda_{i Q}^{G}=2$ times the nonlinear character of $Q$. Therefore $\lambda_{\mid Q}^{G}$ is not multiplicity free.

A result of Brauer, Nesbitt and Osima [1], [7] together with the theorem yield the following

Corollary. $A$ group $G$ has a normal p-complement and abelian $p$ Sylow subgroups if and only if each irreducible character restricted to the p-regular elements is a modular irreducible character. 
Proof. The theorem of Brauer, Nesbitt and Osima says that a group has a normal $p$-complement if and only if each block of $G$ contains exactly one modular character, i.e. if and only if each row of $D$ has only one nonzero entry. Thus if $G$ has a normal $p$-complement and abelian $p$-Sylow subgroups then the nonzero entry in each row must be a 1 by the theorem. Thus each irreducible character is irreducible as a modular character.

Conversely if each character of $G$ is irreducible as a modular character then each row of $D$ consists of 0 's and one 1 . Thus $G$ has a normal $p$-complement. If a $p$-Sylow subgroup $P$ were not abelian then by taking a nonlinear irreducible character of $P$ and extending it to a character $\chi$ of $G$ we would have an irreducible character of $G$ which when viewed as a modular character would decompose into $\chi(1)$ copies of the principal modular character contrary to hypothesis. Thus $P$ is abelian.

\section{REFERENCES}

1. R. Brauer and C. Nesbitt, On the modular characters of groups, Ann. of Math. (2) 42 (1941), 556-590. MR 2, 309.

2. C. W. Curtis and I. Reiner, Representation theory of finite groups and associative algebras, Pure and Appl. Math., vol. 11, Interscience, New York, 1962. MR 26 \#2519.

3. W. Feit, Characters of finite groups, Benjamin, New York, 1967. MR 36 \#2715.

4. P. Fong, On the characters of p-solvable groups, Trans. Amer. Math. Soc. 98 (1961), 263-284. MR 22 \#11052.

5. P. X. Gallagher, Group characters and normal Hall subgroups, Nagoya Math. J. 21 (1962), 223-230. MR 26 \#240.

6. P. Hall and G. Higman, On the p-length of p-soluble groups and reduction theorems for Burnside's problem, Proc. London Math. Soc. (3) 6 (1956), 1-42. MR 17, 344.

7. M. Osima, On primary decomposable group rings, Proc. Phys.-Math. Soc. Japan (3) 24 (1942), 1-9. MR 7, 373.

University of Michigan, Ann Arbor, Michigan 48104 